\title{
Bounds for Value at Risk for Multiasset Portfolios
}

\author{
P. JAWORSKI* \\ Institute of Mathematics, University of Warsaw \\ Banacha 2, 02-097 Warszawa, Poland
}

\begin{abstract}
The theory of copulas provides a useful tool for modeling dependence in risk management. In insurance and finance, as well as in other applications, dependence of extreme events is particularly important, hence there is a need for the detailed study of the tail behaviour of the multivariate copulas. In this paper we investigate the class of copulas being the weighted means of copulas having homogeneous lower tails. We show that having only such information on the structure of dependence of returns from assets is enough to get estimates on value at risk of the multiasset portfolio in terms of value at risk of one-asset portfolios.
\end{abstract}

PACS numbers: 89.65.Gh

\section{Introduction}

The aim of this paper is to show the advantages of modeling the dependence between the extreme events with the help of copulas. Let us consider the following case. An investor operating on an emerging market, has in his portfolio several currencies which are highly dependent. Let $s_{i}, i=1, \ldots, d$ be the quotients of the currency rates at the end and at the beginning of the investment. Let $w_{i}$ be the part of the capital invested in the $i$-th currency, $\sum w_{i}=1, w_{i} \geq 0$. Therefore the final value of the portfolio equals

$$
W_{1}(w)=\left(w_{1} s_{1}+\ldots+w_{d} s_{d}\right) W_{0} .
$$

For portfolio consisting of only one currency (say $i$-th) we put

$$
w=e_{i}=(0, \ldots, 0,1,0, \ldots, 0) .
$$

Let us note that at the moment of the beginning of the investment only $W_{0}$ and $w_{i}$ 's are known. $s_{i}$ 's remain uncertain, therefore we represent them by random variables on a certain probability space $(\Omega, \mathcal{F}, P)$.

The crucial point is to estimate the risk of the investment. As a measure of risk we shall consider "value at risk" (VaR), which in last years became one of the most popular measures of risk in the "practical" quantitative finance (see for

*e-mail: jwptxa@mimuw.edu.pl 
example [1-9]). Roughly speaking the idea is to determine the biggest amount one can lose on a certain confidence level $1-\alpha$ :

$$
\operatorname{VaR}_{1-\alpha}(w)=\sup \left\{V: P\left(W_{0}-W_{1}(w) \leq V\right)<1-\alpha\right\} .
$$

In order to determine $\mathrm{VaR}$ accurately one has to deal with the complexity of the problem. The extremes hardly follow the normal distribution law. Therefore the main challenge is to describe properly the interdependences of risk factors (in our case the changes of currency rates). In this presentation, it will be based on copulas, which are scaleless dependence measures of random variables. We will show that sometimes it is enough to have only the partial information on the given copula.

The main result we would like to present is the diversification formula, i.e.. the following estimate of the value at risk of a given portfolio $w$ in terms of value at risk of one-currency portfolios $e_{i}$ (cf. [10] for two-dimensional case):

$$
\sum w_{i} \operatorname{VaR}_{1-\alpha}\left(e_{i}\right) \geq \operatorname{VaR}_{1-\alpha}(w) .
$$

The above estimate is valid for sufficiently small $\alpha$ under the mild assumptions:

- The copula $C$ of $s_{i}$ 's is a weighted mean (mixture) of copulas $C_{i}$ having nonzero homogeneous lower tails,

$$
\begin{gathered}
C(q)=a_{0} C_{0}(q)+a_{1} C_{1}(q)+\ldots+a_{m} C_{m}(q), \quad a_{0}, \ldots, a_{m} \geq 0, \\
\sum_{i=0}^{m} a_{i}=1
\end{gathered}
$$

and for sufficiently small $q$ :

$$
\begin{aligned}
C_{i}(q) & =L_{i}(q), \quad \forall t>0 \quad L_{i}(t q)=t^{k_{i}} L_{i}(q), \\
1 & =k_{0}<k_{1}<\ldots<k_{m} .
\end{aligned}
$$

- For $i=1, \ldots, d$, for sufficiently small $x$, the function $G_{i}(x)=1 / F_{i}(x)$, where $F_{i}$ is the distribution function of $s_{i}$, is convex (i.e. the hazard rate $F^{\prime}(t) / F(t)^{2}$ is decreasing).

- For $i=1, \ldots, d$, for a positive $w$ and for sufficiently small $\alpha$ :

$$
F_{i}\left(w \cdot\left(F_{1}^{-1}(\alpha)+\ldots F_{d}^{-1}(\alpha)\right)\right) \leq\left\{\begin{array}{ccc}
\delta \alpha^{\frac{1}{k_{1}}} & \text { if } & m \geq 1, \\
\delta & \text { if } & m=0 .
\end{array}\right.
$$

The first assumption is modelling the asymptotic dependence (cf. [11] Th. 2). For example it describes very well the behaviour of foreign exchange rates on an emerging market, where the extreme changes are usually due to the local factors (cf. [10]).

The second one is fulfilled by a wide variety of probability laws. For example it is valid if the distributions of $-\ln s_{i}$ have the same upper tails as normal, Pareto or Gamma distribution (i.e. if their distribution functions coincide for enough big arguments). 
The last one means that the probability distributions of the extreme downfalls for different currencies "behave" in a similar way.

We recall that a function

\section{Notation}

$C:[0,1]^{d} \longrightarrow[0,1]$

is called a copula (see [12] $\S 2.10,[13] \S 4.1,[14] \S 4.4)$ if for every $u=\left(u_{1}, \ldots, u_{d}\right)$ and $v=\left(v_{1}, \ldots, v_{d}\right)\left(u_{i}, v_{i} \in[0,1]\right)$ and every $j \in\{1, \ldots, d\}$

(i) $\quad u_{j}=0 \Rightarrow C(u)=0$;

(ii) $\quad\left(\forall i \neq j u_{i}=1\right) \Rightarrow C(u)=u_{j}$;

(iii) $\quad u \preceq v \Rightarrow V_{C}(u, v) \geq 0$,

where $u \preceq v$ denotes the partial ordering on $\mathbb{R}^{d}\left(u \preceq v \Leftrightarrow \forall i u_{i} \leq v_{i}\right)$ and $V_{C}(u, v)$ is the $C$-volume of the rectangle $I(u, v)$, the one with lower vertex $u$ and upper vertex $v$.

$$
V_{C}(u, v)=\sum_{j_{1}=1}^{2} \ldots \sum_{j_{d}=1}^{2}(-1)^{j_{1}+\ldots+j_{d}} C\left(a_{1, j_{1}}, \ldots, a_{d, j_{d}}\right),
$$

where $a_{i, 1}=u_{i}$ and $a_{i, 2}=v_{i}$ for $i=1, \ldots, d$. The functions with the last property are called $n$-nondecreasing. Those which fulfill the first one are called grounded.

REMARK 1. (cf. [15], Th. 12.5) Every continuous, grounded, $n$-nondecreasing function

$$
H:[0, a]^{d} \longrightarrow \mathbb{R}
$$

is a distribution function of a Borel measure $\mu_{H}$ on $[0, a]^{d}$ :

$$
\begin{aligned}
& H(u)=\mu_{H}(I(0, u)), \\
& \mu_{H}(I(u, v))=\mu_{H}(\operatorname{int}(I(u, v)))=V_{H}(u, v) .
\end{aligned}
$$

Due to the second condition every copula is a distribution function of a probability measure on the unit rectangle $[0,1]^{d}$ with uniform margins (cf. [16], §1.6). Furthermore, the above remark remains true if $H$ is defined on the whole multioctant $[0,+\infty)^{d}$.

Let $\mathcal{X}_{i}, i=1, \ldots, d$ be random variables defined on the same probability space $(\Omega, \mathcal{M}, \mathbb{P})$. Their joint cumulative distribution $F_{\mathcal{X}}$ can be described using an appropriate copula $C_{\mathcal{X}}$ ("Sklar Theorem" see [12], Theorem 2.10.11, [13], Theorem 4.5):

$$
F_{\mathcal{X}}(x)=C_{\mathcal{X}}\left(F_{\mathcal{X}_{1}}\left(x_{1}\right), \ldots, F_{\mathcal{X}_{d}}\left(x_{d}\right)\right),
$$

where $F_{\mathcal{X}_{i}}$ are cumulative distributions of $\mathcal{X}_{i}$. Let us note that the strictly increasing transformations of random variables $\mathcal{X}_{i}$ do not affect the copula. Indeed, if

$$
\mathcal{X}_{i}^{\prime}=f_{i}\left(\mathcal{X}_{i}\right), \quad i=1, \ldots, d
$$

where $f_{i}$ are strictly increasing (and so invertible), then

$$
F_{\mathcal{X}^{\prime}}(x)=F_{\mathcal{X}}\left(f_{1}^{-1}\left(x_{1}\right), \ldots, f_{d}^{-1}\left(x_{d}\right)\right)
$$




$$
=C_{\mathcal{X}}\left(F_{\mathcal{X}_{1}}\left(f_{1}^{-1}(x)\right), \ldots, F_{\mathcal{X}_{d}}\left(f_{d}^{-1}\left(x_{d}\right)\right)\right)=C_{\mathcal{X}}\left(F_{\mathcal{X}_{1}^{\prime}}\left(x_{1}\right), \ldots, F_{\mathcal{X}_{d}^{\prime}}\left(x_{d}\right)\right) .
$$

Therefore if one is interested in tail dependence of random variables rather than in their individual distribution, then the proper choice is to study the copula. The more so, since the copula is uniquely determined at every point $u$ such that the equations $F_{\mathcal{X}_{i}}\left(x_{i}\right)=u_{i}$ have solutions.

For various applications of copulas to finance see for example [13, 17-19, 10, 20].

\subsection{Model assumptions}

We assume that for $t>0$ the distribution function of each $s_{i}-F_{i}(t)$ is positive and the joint probability distribution of $s_{i}$ 's is continuous with respect to the Lebesgue measure and is determined by a copula $C$ :

$$
F_{s}\left(x_{1}, \ldots, x_{d}\right)=C\left(F_{1}\left(x_{1}\right), \ldots, F_{d}\left(x_{d}\right)\right) .
$$

Furthermore, $C$ is a weighted mean of copulas $C_{j}$ :

$$
\begin{gathered}
C(q)=a_{0} C_{0}(q)+a_{1} C_{1}(q)+\ldots+a_{m} C_{m}(q), \\
a_{0}, \ldots, a_{m} \geq 0, \quad \sum_{i=0}^{m} a_{i}=1,
\end{gathered}
$$

and there is a constant $\delta \in(0,1)$ such that:

A1. For $q=\left(q_{1}, \ldots q_{d}\right)$ and $0 \leq q_{i} \leq \delta, C_{j}(q)=L_{j}(q)$, where $L_{j}$ is some nonzero positive homogeneous function of degree $k_{j}, 1=k_{0}<k_{1}<\ldots<k_{m}$ $\left(\forall t>0 \quad L_{j}(t q)=t^{k_{j}} L_{j}(q)\right)$.

A2. For $i=1, \ldots, d$ the function $G_{i}(t)=\frac{1}{F_{i}(t)}$ restricted to $t \in F_{i}^{-1}((0, \delta])$ is convex.

A3. For $i=1, \ldots, d$

$$
\begin{aligned}
\forall w>0 \quad \exists \alpha_{0} \quad \forall 0<\alpha \leq \alpha_{0} \\
\quad F_{i}\left(w \cdot\left(F_{1}^{-1}(\alpha)+\ldots F_{d}^{-1}(\alpha)\right)\right) \leq\left\{\begin{array}{cll}
\delta \alpha^{\frac{1}{k_{1}}} & \text { if } & m \geq 1, \\
\delta & \text { if } & m=0 .
\end{array}\right.
\end{aligned}
$$

The second assumption implies that the preimage of $\delta$ consists of just one point and $F_{i}$ restricted to $\left[0, F_{i}^{-1}(\delta)\right]$ is strictly increasing. Therefore we get a simpler formula for value at risk of one-asset portfolios.

Corollary 1. For $\alpha \in(0, \delta]$,

$$
\operatorname{VaR}_{1-\alpha}\left(e_{i}\right)=W_{0} \cdot\left(1-F_{i}^{-1}(\alpha)\right), \quad i=1, \ldots, d .
$$

Let us note that the above formula is useful for the practical applications. It reduces the determination of $\mathrm{VaR}$ for one-asset portfolios to the estimation of the $\alpha$-quantile of the quotient of the currency rates at the end and at the beginning of the investment, which can be accomplished by a standard statistical procedure.

In $[21,11]$ we showed that there is a large class of copulas whose tails can be approximated by a homogeneous function $L$ of degree 1 . Let us recall the basics about such L's. Comparing [11], Theorem 3, and the construction from the proof 
of Proposition 6 (also [11]) one gets

TheOREM 1. For a homogeneous of degree 1 function $L, L:[0,+\infty)^{d} \rightarrow \mathbb{R}$, the following conditions are equivalent:

1. L is equal to the lower tail of some copula $C$.

2. $L$ is d-nondecreasing and

$$
0 \leq L(u) \leq \min \left(u_{1}, \ldots, u_{d}\right) \quad \text { for } \quad u \succeq 0 .
$$

3. $L$ is continuous, grounded, $d$-nondecreasing and $\mu_{L}=m \times \mu_{\Delta}$, where $m$ is the Lebesgue measure on the real halfline and $\mu_{\Delta}$ is a measure on the unit simplex $\Delta=\left\{q \in \mathbb{R}_{+}^{d}: q_{1}+\ldots+q_{d}=1\right\}$ such that

$$
\int_{\Delta} \frac{1}{q_{i}} \mathrm{~d} \mu_{\Delta}(q) \leq 1 \quad \text { for } \quad i=1, \ldots, d \text {. }
$$

Basing on Theorem 1 we can reduce by one the dimensionality of our problem. Indeed, the multioctant is the Cartesian product of a halfline and simplex, $\mathbb{R}_{+}^{d}=\mathbb{R}_{+} \times \Delta$. Therefore, due to the Fubini Theorem, as a consequence of point 3 of the above theorem, we get the following fact.

Corollary 2. For every closed set $A, A \subset \mathbb{R}_{+}^{d}$,

$$
\mu_{L}(A)=\int_{\Delta} m\left(\mathbb{R}_{+} \xi \cap A\right) \mathrm{d} \mu_{\Delta}(\xi),
$$

where $\mathbb{R}_{+} \xi$ is a halfline spanned by vector $\xi$.

\section{The estimate}

We assume, that $\forall i \quad w_{i}>0$.

TheOREM 2. For $\alpha$ from $(0,1)$, such that

$$
\begin{gathered}
\sum_{i=1}^{d} w_{i} F_{i}^{-1}(\alpha) \leq \min \left\{w_{j} F_{j}^{-1}\left(\delta^{*}\right): j=1, \ldots, d\right\}, \\
\delta^{*}=\left\{\begin{array}{ccc}
\delta \alpha^{\frac{1}{k_{1}}} & \text { if } & m \geq 1, \\
\delta & \text { if } & m=0,
\end{array}\right.
\end{gathered}
$$

the following inequality holds:

$$
\operatorname{VaR}_{1-\alpha}(w) \leq w_{1} \operatorname{VaR}_{1-\alpha}\left(e_{1}\right)+\ldots+w_{d} \operatorname{VaR}_{1-\alpha}\left(e_{d}\right) .
$$

Let us note that due to condition A3 the set of $\alpha$ fulfilling the assumptions of theorem 2 is not empty. The proof of the theorem will be based on the quantile transformation and properties of the following family of sets.

For $\lambda=\left(\lambda_{1}, \ldots, \lambda_{d}\right), \lambda_{i}>0$, we put

$$
Y_{\lambda}=\left\{q \in \mathbb{R}_{+}^{d}: \sum_{i=1}^{d} \frac{\lambda_{i}}{q_{i}} \geq 1\right\} .
$$

LEMMA 1.

Proof.

$\mu_{L_{0}}\left(Y_{\lambda}\right) \leq \sum \lambda_{i}$

We base on the fact that $L_{0}$ is homogeneous of degree 1 and 


$$
\mu_{L_{0}}\left(Y_{\lambda}\right)=\int_{\Delta} m\left(\mathbb{R}_{+} \xi \cap Y_{\lambda}\right) \mathrm{d} \mu_{\Delta}(\xi) .
$$

The intersection of $Y_{\lambda}$ and the halfline given by the vector $\xi$ is a segment of length $\sum \frac{\lambda_{i}}{\xi_{i}}$

$$
\mathbb{R}_{+} \xi \cap Y_{\lambda}=\left\{t: \sum \frac{\lambda_{i}}{t \xi_{i}} \geq 1\right\}=\left\{t: 0 \leq t \leq \sum \frac{\lambda_{i}}{\xi_{i}}\right\}
$$

Therefore

$$
\mu_{L_{0}}\left(Y_{\lambda}\right)=\int_{\Delta} \sum \frac{\lambda_{i}}{\xi_{i}} \mathrm{~d} \mu_{\Delta}(\xi)=\sum \lambda_{i} \int_{\Delta} \sum \frac{1}{\xi_{i}} \mathrm{~d} \mu_{\Delta}(\xi) \leq \sum \lambda_{i} .
$$

For $r>0$ we put

$$
V_{r}=\left\{q \in \mathbb{R}_{+}^{d}: \sum_{i=1}^{d} w_{i} F_{i}^{-1}\left(q_{i}\right) \leq r\right\} \text {. }
$$

Lemma 2. For positive $r$ and $\alpha \in(0,1)$ such that

$$
r=\sum_{i=1}^{d} w_{i} F_{i}^{-1}(\alpha) \leq \min \left\{w_{j} F_{j}^{-1}(\delta): j=1, \ldots, d\right\}
$$

the following inclusions hold:

where

$$
V_{r} \subset\left[0, F_{1}\left(\frac{r}{w_{1}}\right)\right] \times \ldots \times\left[0, F_{d}\left(\frac{r}{w_{d}}\right)\right] \subset[0, \delta]^{d}, \quad V_{r} \subset Y_{\lambda},
$$

$$
\lambda_{i}=\alpha \frac{w_{i} c_{i}^{-1}}{\sum w_{j} c_{j}^{-1}} ; \quad c_{j}=F_{j}^{\prime}\left(F_{j}^{-1}(\alpha)\right) .
$$

Proof.

If $q$ belongs to $V_{r}$ then

$$
\sum_{i=1}^{d} w_{i} F_{i}^{-1}\left(q_{i}\right) \leq r=\sum_{i=1}^{d} w_{i} F_{i}^{-1}(\alpha) \leq \min \left\{w_{j} F_{j}^{-1}(\delta)\right\} .
$$

Therefore for each $i$ :

$$
w_{i} F_{i}^{-1}\left(q_{i}\right) \leq r \leq w_{i} F_{i}^{-1}(\delta)
$$

and

$q_{i} \leq F_{i}\left(\frac{r}{w_{i}}\right) \leq F_{i}\left(F_{i}^{-1}(\delta)\right)=\delta$.

To proof the second inclusion, we use the convexity of $G_{i}=1 / F_{i}$.

$$
\begin{aligned}
\frac{1}{q_{i}}- & \frac{1}{\alpha}=\frac{1}{F_{i}\left(F_{i}^{-1}\left(q_{i}\right)\right)}-\frac{1}{F_{i}\left(F_{i}^{-1}(\alpha)\right)}=G_{i}\left(F_{i}^{-1}\left(q_{i}\right)\right)-G_{i}\left(F_{i}^{-1}(\alpha)\right) \\
& \geq G_{i}^{\prime}\left(F_{i}^{-1}(\alpha)\right)\left(F_{i}^{-1}\left(q_{i}\right)-F_{i}^{-1}(\alpha)\right) \\
& =\frac{-F_{i}^{\prime}\left(F_{i}^{-1}(\alpha)\right)}{\left(F_{i}\left(F_{i}^{-1}(\alpha)\right)\right)^{2}}\left(F_{i}^{-1}\left(q_{i}\right)-F_{i}^{-1}(\alpha)\right)
\end{aligned}
$$




$$
=-\frac{c_{i}}{\alpha^{2}}\left(F_{i}^{-1}\left(q_{i}\right)-F_{i}^{-1}(\alpha)\right)
$$

thus

$$
F_{i}^{-1}\left(q_{i}\right)-F_{i}^{-1}(\alpha) \geq-\frac{\alpha^{2}}{c_{i}}\left(\frac{1}{q_{i}}-\frac{1}{\alpha}\right) .
$$

If $q$ belongs to $V_{r}$ then we obtain

$$
\begin{aligned}
0 \geq & \sum_{i=1}^{d} w_{i} F_{i}^{-1}\left(q_{i}\right)-r=\sum_{i=1}^{d} w_{i} F_{i}^{-1}\left(q_{i}\right)-\sum_{i=1}^{d} w_{i} F_{i}^{-1}(\alpha) \\
& \geq-\sum_{i=1}^{d} \frac{w_{i} \alpha^{2}}{c_{i}}\left(\frac{1}{q_{i}}-\frac{1}{\alpha}\right) \\
& =-\alpha\left(\sum_{i=1}^{d} \frac{\lambda_{i}}{q_{i}} \sum_{j=1}^{d} \frac{w_{j}}{c_{j}}-\sum_{i=1}^{d} \frac{w_{i}}{c_{i}}\right)=-\alpha \sum_{j=1}^{d} \frac{w_{j}}{c_{j}}\left(\sum_{i=1}^{d} \frac{\lambda_{i}}{q_{i}}-1\right) .
\end{aligned}
$$

Therefore

$$
0 \leq \sum \frac{\lambda_{i}}{q_{i}}-1
$$

and therefore $q$ belongs to $Y_{\lambda}$.

Corollary 3. Under the assumptions of theorem 2 and lemma 2, for each $i$ $\mu_{L_{i}}\left(V_{r}\right) \leq \alpha$.

Proof.

Case $L_{0}$. We base on the inclusion $V_{r} \subset Y_{\lambda}$ (lemma 2) and the estimated measure of $Y_{\lambda}$ (lemma 1).

$$
\mu_{L_{0}}\left(V_{r}\right) \leq \mu_{L_{0}}\left(Y_{\lambda}\right) \leq \sum \lambda_{i}=\alpha .
$$

Case $L_{i}, i=1, \ldots, m$. We base on the inclusion $V_{r} \subset \times_{i=1}^{d}\left[0, F_{i}\left(\frac{r}{w_{i}}\right)\right]$ from lemma 2 and the quasihomogeneity of $L_{i}$.

$$
\begin{gathered}
\mu_{L_{i}}\left(V_{r}\right) \leq \mu_{L_{i}}\left(\times_{i=1}^{d}\left[0, F_{i}\left(\frac{r}{w_{i}}\right)\right]\right)=L_{i}\left(F_{1}\left(\frac{r}{w_{1}}\right), \ldots, F_{d}\left(\frac{r}{w_{d}}\right)\right) \\
\leq L_{i}\left(\delta \alpha^{\frac{1}{k_{1}}}, \ldots, \delta \alpha^{\frac{1}{k_{1}}}\right)=\alpha^{\frac{k_{i}}{k_{1}}} L_{i}(\delta, \ldots, \delta)=\alpha^{\frac{k_{i}}{k_{1}}} C_{i}(\delta, \ldots, \delta) \leq \alpha .
\end{gathered}
$$

Proof of theorem 2.

In order to estimate $\operatorname{VaR}_{1-\alpha}(w)$ we consider

$$
\begin{aligned}
1- & P\left(W_{0}-W_{1}(w) \leq \sum w_{i} \operatorname{VaR}_{1-\alpha}\left(e_{i}\right)\right) \\
& =P\left(W_{0}-W_{1}(w) \geq \sum w_{i} \operatorname{VaR}_{1-\alpha}\left(e_{i}\right)\right) \\
& =P\left(1-\sum w_{i} s_{i} \geq \sum w_{i}\left(1-F_{i}^{-1}(\alpha)\right)\right) \\
& =P\left(\sum w_{i} s_{i} \leq \sum w_{i} F_{i}^{-1}(\alpha)\right)
\end{aligned}
$$


Therefore

$$
\begin{aligned}
& =P\left(\sum w_{i} s_{i} \leq r\right)=\mu_{C}\left(V_{r}\right) \\
& =\sum a_{i} \mu_{C_{i}}\left(V_{r}\right)=\sum a_{i} \mu_{L_{i}}\left(V_{r}\right) \leq \sum a_{i} \alpha=\alpha .
\end{aligned}
$$

$$
P\left(W_{0}-W_{1}(w) \leq \sum w_{i} \operatorname{VaR}_{1-\alpha}\left(e_{i}\right)\right) \geq 1-\alpha .
$$

Since

$$
\operatorname{VaR}_{1-\alpha}(w)=\sup \left\{V: P\left(W_{0}-W_{1}(w) \leq V\right)<1-\alpha\right\}
$$

we obtain the estimate

$$
\operatorname{VaR}_{1-\alpha}(w) \leq \sum w_{i} \operatorname{VaR}_{1-\alpha}\left(e_{i}\right)
$$

REMARK 2. If $a_{0}=1$ then condition A3 is not necessary (cf. [20]), on the other hand if $a_{0}=0$ then we may omit condition A2.

\section{Conclusions}

We proved in this paper that under the mild assumptions, for sufficiently small $\alpha$ the value at risk of a portfolio such that $w_{i}$ part of the capital is invested in $i$-th currency $\left(w_{i} \geq 0\right)$ is smaller the $w$-weighted sum of values at risk of oneasset portfolios

$$
\operatorname{VaR}_{1-\alpha}(w) \leq w_{1} \operatorname{VaR}_{1-\alpha}\left(e_{1}\right)+\ldots+w_{d} \operatorname{VaR}_{1-\alpha}\left(e_{d}\right)
$$

Since the supervision institutions, like the Basle Committee on Banking Supervision (cf. [1]), impose the rules that the value of risk should not exceed certain threshold, such an estimation simplifies the task of a risk manager. Indeed, if the $w$-weighted sum of VaR's of one-asset portfolios does not exceed the threshold so does the VaR of the portfolio $w$.

\section{References}

[1] Basle Committee on Banking Supervision, Amendment to the Capital Accord to Incorporate Market Risks, Basle 1996.

[2] Risk Metrics - Technical Document, 1996, Morgan Guaranty Trust Company of New York.

[3] CreditMetrics — Technical Document, 1997, J.P. Morgan and Co. Incorporated.

[4] H. Föllmer, A. Schied, Stochastic Finance. An Introduction in Discrete Time, de Gruyter, Berlin 2004, p. 470.

[5] P.J. Cumperayot, J. Danielsson, B.J. Jorgensen, C.G. de Vries, in: Measuring Risk in Complex Stochastic Systems, Eds. J. Franke, W. Härdle, G. Stahl, Lecture Notes in Statistics, Vol. 147, Springer, New York 2000, p. 257.

[6] A.J. McNeil, in: Extremes and Integrated Risk Management, Ed. P. Embrechts, Risk Waters Group Ltd, London 2000, p. 397.

[7] F.M. Longin, J. Banking Finance 24, 1097 (2000).

[8] P. Jackson, D.J. Maude, W. Perraudin, J. Derivatives Spring, 73 (1997). 
[9] G.C. Pflug, in: Probabilistic Constrained Optimization: Methodology and Applications, Ed. S. Uryasev, Kluwer Academic Publ., 2000, p. 320.

[10] P. Jaworski, Acta Phys. Pol. B 37, 3005 (2006).

[11] P. Jaworski, Appl. Math. 33, 159 (2006).

[12] R.B. Nelsen, An Introduction to Copulas, Springer, New York 1999, p. 230.

[13] U. Cherubini, E. Luciano, W. Vecchiato, Copula Methods in Finance, Wiley, Hoboken 2004, p. 310.

[14] C. Alsina, M. Frank, B. Schweizer, Associative Functions: Triangular Norms and Copulas, World Sci., New Jersey 2006, p. 252.

[15] P. Billingsley, Probability and Measure, Wiley, New York 1979, p. 600.

[16] H. Joe, Multivariate Models and Dependence Concepts, Chapman and Hall, London 1997, p. 424.

[17] P. Embrechts, A. Höing, J. Alessandro, Finance Stoch. 7, 145 (2003).

[18] P. Embrechts, G. Puccetti, Finance Stoch. 10, 341 (2006).

[19] P. Jaworski, Acta Phys. Pol. B 36, 2575 (2005).

[20] P. Jaworski, in: Proc. 5th EUSFLAT Conf., Ostrava (Czech Republic) 200\%, Eds. M. Stepnicka, V. Novak, U. Bodenhofer, University of Ostrava, Ostrava 2007, Vol. 1, p. 500.

[21] P. Jaworski, Appl. Math. 31, 397 (2004). 